\title{
Should general practitioners order troponin tests?
}

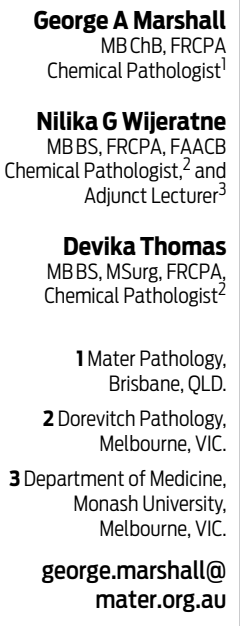

doi: 10.5694/mjal3.00173

Editorial $p 125$ Research $p 158$
C ardiac troponin I and T are the preferred biomarkers for assessing myocardial injury. Understanding the pathobiology of troponin and the timing of troponin testing is fundamental to the clinical utility of these biomarkers, as troponin and its kinetics are central to the universal definition of acute myocardial infarction (AMI). ${ }^{1}$ Troponin levels become elevated in serum within a few hours of an AMI, and they remain elevated for up to 7-10 days. ${ }^{2}$ However, numerous other conditions may elevate troponin levels, so it remains essential that the results of troponin tests be interpreted with clinical findings and electrocardiography results. ${ }^{3}$ The dynamics of troponin levels (rise and/or fall over time) help distinguish AMI from non-AMI conditions, thus serial troponin testing is the standard approach recommended for assessing patients with suspected acute coronary syndrome (ACS). ${ }^{4}$ In this article, we explore troponin testing in general practice, including problems faced by laboratories that offer testing in this context.

\section{The absolutist stance}

One stance on this topic is that general practitioners should never order a troponin test. The basis of this argument is that the only widely accepted clinical indication for measurement of troponin levels is suspected ACS, which should prompt referral to hospital based on clinical and electrocardiography findings without recourse to troponin testing. Supporting this argument is that serial troponin testing is unrealistic in most general practice settings, and opens the question of how a patient should be monitored while the results are awaited.

One argument against this absolutist stance is that it is an oversimplification. Further investigation and management depends on the degree of suspicion for ACS, and timing of the presentation may obviate the need for serial testing. Chest pain is a challenging symptom and the prevalence of unstable angina or AMI in general practice is low, in the order of fewer than $5 \%$ of patients with chest pain. ${ }^{5}$ Atypical presentations of AMI, such as in young people, ${ }^{6}$ people with diabetes and older people, are a perennial concern. GPs have been shown to be fairly accurate in assessing chest pain clinically as due to coronary artery disease, but not accurate enough to safely exclude it. ${ }^{7}$ A system of estimating pretest probability of ACS, or risk of short-term complications, is an attractive approach. Clinical decision-making rules and pretest probability tables have been developed to assist with this process in general practice ${ }^{8,9}$ and, while some risk stratification tools may be more relevant to doctors in emergency departments, they are potentially useful to GPs. ${ }^{10,11}$ How troponin testing might fit into risk stratification in general practice is not entirely clear.

\begin{abstract}
Summary
Cardiac troponin I and T are the preferred biomarkers for assessing myocardial injury, and the timing of troponin testing is fundamental to its clinical utility.

- There are arguments for and against the use of troponin testing in the community, and the stance that general practitioners should never order a troponin test can be considered an oversimplification.

- GPs have a generally sufficient understanding of the test for use in primary care, and have a better understanding of false-negative troponin test results than falsepositive results.

We suggest that hospitalisation, rather than troponin testing, should be the default option for patients with symptoms suggestive of acute coronary syndrome.

- A single troponin test is reasonable in primary care to exclude the possibility of acute myocardial infarction in asymptomatic low-risk patients whose symptoms resolved at least 12 hours prior.

GPs should factor in the complex logistics of troponin testing in the community before ordering a troponin test: results need to be accurate and timely, and might be obtained at a time of day when it is difficult to contact the doctor or the patient.
\end{abstract}

\section{How well do GPs understand troponin tests?}

Audits from New Zealand suggest that GPs have a generally sufficient understanding of the use of troponin tests in primary care. ${ }^{12,13}$ Knowledge of false-negative results (eg, due to sampling too soon after symptoms) appears to be better than knowledge of false-positive results (eg, due to non-AMI causes of raised troponin levels). Most GPs would refer high-risk patients without troponin testing, but a small proportion of GPs would defer hospitalisation while waiting for the troponin result (mostly for patients with an intermediate probability of AMI).

\section{Why do GPs request troponin tests?}

Our experience suggests that GPs mainly request troponin tests to rule out AMI in one of two situations. The first situation is patients who had symptoms in the preceding days but for whom symptoms have resolved (also the experience of others $\left.{ }^{13,14}\right)$. One expert has suggested that this may be a justifiable use of troponin testing in primary care ${ }^{15}$ and troponin testing is suggested in National Institute for Health and Care Excellence (NICE) primary care guidelines in pain-free patients who had chest pain more than 72 hours earlier. ${ }^{9}$ The second situation is patients who have atypical symptoms and/or a low likelihood of ACS, in whom troponin testing appears to cover the residual clinical uncertainty. Unexpectedly positive troponin results occasionally occur in such situations, which may otherwise not be detected. 


\section{Suggestions for using, or not using, troponin tests in general practice}

\section{The default position}

- The default position for patients who have symptoms suggestive of acute coronary syndrome is hospitalisation without prior troponin testing.

\section{Using a single troponin test}

- It is reasonable to use a single troponin test in general practice to exclude the possibility of acute myocardial infarction (AMI) in asymptomatic patients whose symptoms (typical or otherwise) resolved at least 12 hours prior, so long as they have no high-risk features and a normal electrocardiogram."

- A single troponin test may also be useful to investigate an otherwise unexplained creatine kinase elevation.

Using serial troponin tests

- In patients presenting to general practice within 12 hours of symptom onset who are at low risk of AMl and/or have atypical symptoms, and for whom troponin testing is being considered, serial testing is advised.

- In patients with conditions that are associated with a high prevalence of positive troponin test results, such as chronic kidney disease, a single test can be misleading. Serial testing may be required to resolve clinical uncertainty.

- Serial testing is most appropriately performed in hospital. The safety of serial testing in outpatient settings has not been established.

\section{How do GPs currently request troponin tests?}

Most requests for troponin testing from general practice are requests for a single test, not serial testing. ${ }^{16}$ This begs the question of whether ordering a single troponin test is an appropriate strategy. Given our understanding of troponin kinetics, a single negative troponin test result a certain time after symptom onset could be clinically useful in ruling out AMI (ie, in "late presenters"). The suggested time frame varies between publications, but is usually in the order of 6-9 hours ${ }^{4,17}$ with the caveat that the time of symptom onset can be unreliable. Local experts have suggested that a single troponin test 12 hours after resolution of suggestive symptoms (with a normal electrocardiogram and no high-risk features) is useful for this purpose. ${ }^{11}$ With the so-called high-sensitivity troponin assays, this window may decrease: in an emergency department setting, an undetectable (ie, not merely negative) troponin value obtained from a high-sensitivity troponin assay at presentation has been shown to have a very high negative predictive value for a subsequent diagnosis of AMI, ${ }^{18}$ but this strategy is experimental. The safest rule of thumb is that a single negative test result for troponin does not exclude AMI in a patient with current or very recent symptoms, nor does it exclude clinically significant coronary artery disease.

\section{Conditions associated with chronic troponin elevation}

As most GP requests for troponin testing are for a single test, conditions associated with chronic, non-AMI elevation of troponin levels present a problem. Examples include chronic cardiac failure and chronic kidney disease (CKD). A positive result from a single troponin test could be misleading because it might reflect the underlying chronic disease and not AMI. The prevalence of positive troponin test results (defined as above the 99th percentile of the general population) in CKD depends on the stage of the CKD (positive results are more likely during more advanced stages) and on the troponin assay used. This is exemplified by a recent study of asymptomatic patients who had CKD but were not on dialysis. The prevalence of a positive troponin result (for the whole cohort) was $68 \%$ when a high-sensitivity troponin T assay was used, 38\% when a high-sensitivity troponin I assay was used, and 16\% and 8\% for troponin T and I, respectively, when contemporary ("less sensitive") assays were used. ${ }^{19}$ Despite the high rates of positive troponin results in this study, a negative troponin result from a sample taken at an appropriate time is useful for ruling out AMI in patients who have CKD, but at the considerable disadvantage of reduced positive predictive value, with the attendant risk of unnecessary hospitalisation. Clinical assessment of the acute event in such patients becomes all the more important if this is to be avoided.

\section{Logistics of troponin testing for outpatients}

Offering troponin testing in the community is logistically complex and there is a lack of formal guidance for laboratories in this area. Guidelines on management of ACS recommend that a troponin test result should be available within 60 minutes of blood being drawn and, if not, that point-of-care testing should be available. ${ }^{4}$ This is aimed at hospital-based laboratories and is not a realistic target for large private pathology networks that may test hundreds of community samples per day at variable geographical distances from large networks of collection centres and general practices. So what is the solution? Accept the longer turnaround times and promote judicious use of troponin tests by GPs? Longer turnaround times may be acceptable if testing is largely confined to patients who have a low pretest probability, or low risk, of AMI. If so, what is a reasonable turnaround time for community samples three hours? Six? At the other extreme is rigorous pursuit of fast turnaround times to meet the apparent clinical need in the community, probably with the help of point-of-care testing, although there are questions about the performance of point-of-care troponin assays. ${ }^{20}$ The solution is probably a compromise between the two. The only guidelines that provide advice on this are the NICE guidelines, which state that troponin testing can be undertaken in general practice "providing timely results can be obtained" but do not elaborate on what "timely" means. ${ }^{9}$

After-hours elevated troponin levels can be problematic for all concerned. For example, when samples are taken late in the afternoon, results might not be available until after clinic hours. A common policy is to treat positive troponin test results as "critical results" and to notify the requesting doctor or a representative (such as a locum GP service, if nominated). In the event that a doctor cannot be found to take the result, which is not uncommon in our experience, laboratory staff (usually pathologists) phone patients directly and advise that hospitalisation is the safest course of action. But when a patient cannot be contacted, laboratory staff face a dilemma: can the result wait until office hours, or should emergency services be arranged? We are aware of anecdotal cases in which after-hours notifications of high troponin levels to patients at home have probably contributed to their early survival — but this raises the question of whether such patients are better served by referral to hospital in the first instance. A published coroner's case touches on these important issues for both GPs and pathologists. ${ }^{21}$ 


\section{Conclusion}

We suggest that GPs should have a high threshold for requesting troponin testing and carefully assess risk before ordering troponin tests. Positive troponin test results usually change the course of management, but the time frame in which the result becomes available must be balanced against the risk of delay in diagnosis and therapy. A troponin test should not be requested unless a GP is certain that a robust process is in place by which they can be contacted, day or night, if the result is positive. There is an obvious need for further education, research and inclusion of this topic in future clinical guidelines. Our suggestions for using, or not using, troponin tests in general practice are summarised in the Box.

Competing interests: No relevant disclosures.

Provenance: Not commissioned; externally peer reviewed.

1 Thygesen K, Alpert JS, Jaffe AS, et al. Third universal definition of myocardial infarction. J Am Coll Cardiol 2012; 60: 1581-1598.

2 Eriksson S, Wittfooth S, Pettersson K. Present and future biochemical markers for detection of acute coronary syndrome. Crit Rev Clin Lab Sci 2006; 43: 427-495.

3 Kelley WE, Januzzi JL, Christenson RH. Increases in cardiac troponin in conditions other than acute coronary syndrome and heart failure. Clin Chem 2009; 55: 2098-2112.

4 Chew DP, Aroney CN, Aylward PE, et al. 2011 Addendum to the National Heart Foundation of Australia/Cardiac Society of Australia and New Zealand Guidelines for the management of acute coronary syndromes (ACS) 2006. Heart Lung Circ 2011; 20: 487-502.

5 Bösner S, Becker A, Haasenritter J, et al. Chest pain in primary care: epidemiology and pre-work-up probabilities. Eur J Gen Pract 2009; 15: 141-146.

6 Dwivedi G, Hayat SA, Violaris AG, Senior R. A 28 year old postpartum woman with right sided chest discomfort: case outcome. BMJ 2006; 332: 643.
7 Nilsson S, Ortoft K, Mölstad S. The accuracy of general practitioners' clinical assessment of chest pain patients. Eur J Gen Pract 2008; 14: 50-55.

8 Bruins Slot MH, Rutten FH, van der Heijden GJ, et al. Diagnosing acute coronary syndromes in primary care: comparison of the physicians' risk estimation and a clinical decision rule. Fam Pract 2011; 28: 323-328.

9 Smeeth L, Skinner JS, Ashcroft J, et al. NICE clinical guideline: chest pain of recent onset. Br J Gen Pract 2010; 60: 607-610.

10 Acute Coronary Syndrome Guidelines Working Group. Guidelines for the management of acute coronary syndromes 2006. Med J Aust 2006; 184 (8 Suppl): S9-S29.

11 Parsonage WA, Cullen L, Younger JF. The approach to patients with possible cardiac chest pain. Med J Aust 2013; 199: 30-34.

12 Law K, Elley R, Tietjens J, Mann S. Troponin testing for chest pain in primary healthcare: a survey of its use by general practitioners in New Zealand. NZ Med J 2006; 119: U2082.

13 Mann S, Tietjens J, Law K, Elley R. Troponin testing for chest pain in primary healthcare: a New Zealand audit. N Z Med J 2006; 119: U2083.

14 Sodi R, Hine T, Shenkin A. General practitioner (GP) cardiac troponin test requesting: findings from a clinical laboratory audit. Ann Clin Biochem 2007; 44: 290-293.

15 Collinson P. The role of cardiac troponin measurements. GP [internet] 2010; 11 Mar. http://www.gponline.com/Clinical/article/988621/rolecardiactroponin-measurements (accessed Nov 2013).

16 Aldous S, Ghent P, McGeoch G, Nicholson D. The use of troponin in general practice. NZ Med J 2012; 125: 36-43.

17 Thygesen K, Mair J, Katus H, et al. Recommendations for the use of cardiac troponin measurement in acute cardiac care. Eur Heart J 2010; 31: 2197-2204.

18 Body R, Carley S, McDowell G, et al. Rapid exclusion of acute myocardial infarction in patients with an undetectable troponin using a high-sensitivity assay. J Am Coll Cardiol 2011; 58: 1332-1339.

19 deFilippi C, Seliger SL, Kelley W, et al. Interpreting cardiac troponin results from high-sensitivity assays in chronic kidney disease without acute coronary syndrome. Clin Chem 2012; 58: 1342-1351.

20 Palamalai V, Murakami MM, Apple FS. Diagnostic performance of four point of care cardiac troponin I assays to rule in and rule out acute myocardial infarction. Clin Biochem 2013; 46: 1631-1635.

21 Bird S. Follow up of test results after hours. Aust Fam Physician 2007; 36 : 761-762. 